\title{
Evaluation of MAGE-A expression and grade of dysplasia for predicting malignant progression of oral leukoplakia
}

\author{
JUTTA RIES $^{1}$, ABBAS AGAIMY ${ }^{2}$, ELEFTERIOS VAIRAKTARIS ${ }^{3}$, YEEUN KWON ${ }^{1}$, \\ FRIEDRICH W. NEUKAM ${ }^{1}$, LEI H. STRASSBURG ${ }^{1}$ and EMEKA NKENKE ${ }^{1}$ \\ ${ }^{1}$ Department of Oral and Maxillofacial Surgery and ${ }^{2}$ Institute of Pathology, Erlangen University Hospital, \\ Erlangen, Germany; ${ }^{3}$ Department of Oral and Maxillofacial Surgery, University of Athens, Athens, Greece
}

Received March 16, 2012; Accepted May 17, 2012

DOI: $10.3892 /$ ijo.2012.1532

\begin{abstract}
The risk of the malignant transformation of oral leukoplakia (OLP) is difficult to predict by histopathology. Melanoma-associated antigen-A (MAGE-A) expression is restricted to malignant cells and may be useful for the more accurate estimation of the potential malignant transformation of pre-malignant lesions. The aim of the present study was to investigate whether the expression of MAGE-A can be used to predict the malignant transformation of OLP. Paraffinembedded tissue samples of OLP from 74 patients followed-up for at least 5 years were included. A total of 24 progressing and 50 non-progressing OLP, 18 corresponding tumor and 30 healthy mucosa specimens were analysed for MAGE-A 1, 3, 4, 610 and 12 expression by nested real-time RT-PCR and graded for dysplasia. In total, $46 \%$ of the progressing lesions expressed at least 1 out of the examined MAGE-A antigens, whereas no expression was detected in any of the non-progressing OLP and normal specimens. The correlation between malignant transformation and MAGE-A expression was statistically significant ( $\mathrm{p}=0.00001$ ). Furthermore, $42 \%$ of the progressing OLPs without dysplasia (D0) expressed at least 1 antigen. The correlation between the grade of dysplasia and MAGE-A staining in the malignant transformation group was not significant $(\mathrm{p}=0.08)$. The detection of at least 1 MAGE-A antigen may allow the identification of high-risk lesions that may progress into carcinoma with time. Therefore, the investigation of MAGE-A expression should be assessed in order to obtain a more accurate evaluation of the potential cancer risk of OLP.
\end{abstract}

Correspondence to: Dr Jutta Ries, Department of Oral and Maxillofacial Surgery, Erlangen University Hospital, Glueckstrasse 11, D-91054 Erlangen, Germany

E-mail: jutta.ries@uk-erlangen.de

Key words: melanoma-associated antigen-A expression, real-time RT-PCR, risk assessment of oral leukoplakia, oral squamous cell carcinoma

\section{Introduction}

It is commonly accepted, that the detection of oral squamous cell carcinoma (OSCC) at an early stage improves the 5-year survival rate to $30 \%$ for patients suffering from advanced stages to approximately $85 \%$ in the early stages. Currenlty, approximately two thirds of the patients are diagnosed in an advanced stage (1-3). Moreover, not all OSCCs develop de novo on healthy mucosal tissue but are often preceded by potentially precancerous lesions and the prognostic interpretation of these lesions may have an impact on early diagnosis (4). Therefore, the early diagnosis and identification of high-risk pre-malignant lesions of the oral mucosa is the most effective approach for reducing morbidity and mortality.

In particular, the estimation of the risk of malignant transformation of oral leukoplakia (OLP), which is the most common potentially malignant disorder of the oral cavity, is challenging (5). At present, the gold standard of the classification and the assessment of patient risk for the development of cancer include a histopathological examination of incision biopsies and the scoring of dysplasia, as it is generally accepted that cancer risk increases with the severity of dysplasia $(6,7)$. Thus, this criterion is commonly used to predict the behaviour of these lesions and to plan further clinical treatment. However, the assessment of dysplasia is highly subjective and lacks sensitivity for the prediction of the behaviour of lesions. It is evident that incision biopsies have limited reproducibility within the whole lesion and several studies have shown great interexaminer and intraexaminer variability in the assessment of the presence or absence and the grade of oral epithelial dysplasia (6-13). It has been demonstrated that OLPs which transform into cancerous lesions clearly harbor genetic instability and accumulate genetic alterations and that these differences are reflected in the oral mucosa by a series of well-defined clinical and histological changes in concordance to dysplasia. Therefore, molecular biological changes in premalignant lesions will hopefully provide a basis for a more accurate prediction and prognosis. However, to date, none of these new markers either alone or in combination are ready for clinical diagnosis and identification of high-risk lesions Therefore, the development of more objective molecular tools remains crucial $(9,10,14-20)$. 
The expression of the melanoma-associated antigen-A (MAGE-A) is restricted to the testis and placenta tissues and various tumors, including $\operatorname{OSCC}(21,22)$. Furthermore, at least 1 MAGE-A gene out of the 10 members belonging to this family can be detected by RT-PCR analyses in $93 \%$ of OSCC cases, but not in healthy normal oral mucosa, making these genes attractive tools for the detection of cancer cells and early diagnosis (23). Moreover, their expression has been detected in potentially pre-cancerous oral lesions and may be useful in discerning high-risk cancerous lesions (24-26). However, these studies had certain limitations, including the fact that only case studies were performed, clinical and histological characteristics were heterogeneous and there was no clinical follow-up. In addition, only a few lesions with no dysplasia were included and clinical outcome was not documented for any of these. Thus, the value of MAGE-A expression analyses in OLP has to be validated by studies including a greater number of cases, particularly D0-OLPs, and by clinical follow-up studies.

The aim of the present study was to analyse the expression of MAGE-A by highly sensitive nested real-time RT-PCR analyses in order to investigate whether the detection of at least 1 MAGE-A antigen out of 6 in OLP can predict the development of OSCC within 5 years. Our results should help clarify whether these molecular parameters can aid in the early detection of tumor cells in suspicious oral lesions or in the prediction of the potentially malignant transformation of OLP.

\section{Materials and methods}

Patients and tissue samples. For the analysis of MAGE-A expression, we used 122 formalin embedded samples separated into 4 groups. The study was approved by the Ethics Committee of the University of Erlangen-Nuremberg and patient informed consent was obtained. A total of 74 OLP samples were analysed that had been collected between 1997 and 2004. These samples were divided into 2 groups and 2 subgroups: Group 1 included 24 OLP samples of patients who developed OSCC during the 5-year follow-up. From these patients, 18 corresponding OSCC samples (group 4) were available. Group 2 consisted of 50 patients, who did not present with malignant transformation of OLP during the 5-year follow-up. A total of 30 samples of normal oral mucosa from healthy volunteers (group 3) served as the negative control. Samples of testis tissue were used as the positive control for MAGE-A expression. Clinical information was collected, including age, gender and time interval between the diagnosis of OLP and the onset of malignancy [disease-free survival time (DFS)].

Oral epithelial dysplasia classification and tumor histopathology and staging. All biopsies were evaluated by 2 pathologists to ensure consistent results. The grade of oral epithelial dysplasia in the OLP samples was histopathologically classified according to the WHO classification, 2005. Based on certain architectural, histological and cytological features, dysplasia was divided into 4 stages: D0, no dysplasia; D1, mild; D2, moderate; and D3, severe dysplasia (9). Ideally, diagnosis corresponds to the nature of the lesion meaning that the level of epithelial dysplasia implies the risk of progression into malignancy (low risk; moderate or severe risk of malignancy) (7).
Clinical staging and TNM classification were carried out for each tumor patient developing a malignancy based on the primary precursor lesion according to the Union for International Cancer Control (UICC). The OSCCs were also classified according to the WHO criteria for differentiation: G1, well-differentiated; G2, moderately; and G3, poorly differentiated. Clinical staging (stages I to IV) and classification according to early (including stages I and II) and late (including stages III and IV) clinical stages were recorded.

RNA isolation and expression analysis of MAGE-A by nested real-time RT-PCR analysis. For expression analyses, the highly sensitive nested RT-PCR method was used for the detection of MAGE-A1, 3, 4, 6, 10 and 12 expression. In summary, total RNA was isolated from paraffin-embedded tissues using the RNeasy FFPE kit (Qiagen, Hilden, Germany) according to the instructions of the manufacturer. RNA quality and quantity were assessed by the 'NanoDrop1000' (peQLab Technologies, Erlangen, Germany) according to the manufacturer's instructions. A total of $200 \mathrm{ng}$ of total RNA was examined for MAGE-A gene expression.

Reverse transcription and the first PCR reaction were carried out using the One-Step RT-PCR kit (Qiagen) and outer primers which were specific for the individual MAGE-A member. For the detection of MAGE-A 3/6 a common primer set was applied (27) (Table I). Cycling conditions were as follows: Reverse transcription was carried out at $50^{\circ} \mathrm{C}$ for $30 \mathrm{~min}$. Initial PCR activation step was performed at $95^{\circ} \mathrm{C}$ for $15 \mathrm{~min}$, followed by 30 cycles of $94^{\circ} \mathrm{C}$ for $30 \mathrm{sec}, 60^{\circ} \mathrm{C} / 58^{\circ} \mathrm{C}$ for $45 \mathrm{sec}$, and $72^{\circ} \mathrm{C}$ for $60 \mathrm{sec}$. The final extension incubation was performed at $72^{\circ} \mathrm{C}$ for $10 \mathrm{~min}$.

To exclude false-positive results generated by the amplification of genomic DNA sequences that were not totally eliminated by DNase digestion, $1 \mu \mathrm{l}$ of purified RNA from each specimen was tested for the amplification of genomic GAPDH using the specific primers for PCR. Only RNA isolations showing no visible band on a $2 \%$ agarose gel were analysed for subsequent procedures.

All first PCR products were diluted (1:10; MAGE-A10 and GAPDH 1:100) and $1 \mu \mathrm{l}$ of these solutions was used as the template for second (nested) PCR. The detection of mRNA was performed with the ABI Prism 7300 Sequence Detection System (Applied Biosystems, Weiterstadt, Germany). The QuantiTect TM SYBR ${ }^{\circledR}$-Green PCR kit (Cat. 204143; Qiagen) and gene-specific nested primers (Table I) were used for PCR amplification. The cycling conditions applied for the nested real-time PCR were as follows: Initial denaturation/enzyme activation for $15 \mathrm{~min}$ at $95^{\circ} \mathrm{C}$ followed by 40 cycles of denaturation at $94^{\circ} \mathrm{C}$ for $15 \mathrm{sec}$, annealing/elongation reaction at $60^{\circ} \mathrm{C}$ for $1 \mathrm{~min}$. Each RT-PCR experiment was performed in duplicate using the same RNA sample and the appropriate controls. The production of a single product and the formation of undesired side products during PCR that contribute to fluorescence were assessed by melting curve analysis. For normalisation and as control for RNA integrity, the amplification of GAPDH was used.

Statistical analysis. Statistical analysis was performed using the statistical software package SPSS 17 (SPSS Inc. Chicago, IL, USA). The prevalence of MAGE-A expression in OLP with 
Table I. Sequences of oligonucleotide primers for the nested RT-PCR amplification of the different MAGE-A genes and the internal control, GAPDH.

\begin{tabular}{|c|c|c|c|c|}
\hline Primer & Sequence ( $5^{\prime}$ to $\left.3^{\prime}\right)$ & Primer (bp) & Amplicon (bp) & Annealing temperature $\left({ }^{\circ} \mathrm{C}\right)$ \\
\hline \multicolumn{5}{|l|}{ One-step RT-PCR } \\
\hline MAGE-A1 o/s & GTA GAG TTC GGC CGA AGG ACC & 21 & 150 & 58 \\
\hline MAGE-A1 o/as & CAG GAG CTG GGC AAT GAA GAC & 21 & & \\
\hline MAGE-A3/6 o/s & GAA GCC GGC CCA GGC TCG & 18 & 151 & 60 \\
\hline MAGE-A3/6 o/as & GAT GAC TCT GGT CAG GGC AA & 20 & & \\
\hline MAGE-A4 o/s & CAC CAA GGA GAA GAT CTG CCT & 21 & 185 & 58 \\
\hline MAGE-A4 o/as & TCC TCA GTA GTA GGA GCC TGT & 21 & & \\
\hline MAGE-A10 o/s & CTA CAG ACA CAG TGG GTC GC & 20 & 139 & 60 \\
\hline MAGE-A10 o/as & GCT TGG TAT TAG AGG ATA GCA G & 22 & & \\
\hline MAGE-A12 o/s & CGC CGC TTT AAC CGC AGG GA & 20 & 181 & 60 \\
\hline MAGE-A12 o/as & CCT GTC TCC TCA GAA CCT GGA TGC T & 25 & & \\
\hline GAPDH o/s & GAC CCC TTC ATT GAC CTC AAC TA & 23 & 102 & 60 \\
\hline GAPDH o/as & TGA CAA GCT TCC CGT TCT CA & 20 & & \\
\hline \multicolumn{5}{|l|}{ Real-time PCR } \\
\hline MAGE-A1 i/s & TAG AGT TCG GCC GAA GGA AC & 20 & 143 & 60 \\
\hline MAGE-A1 i/as & CTG GGC AAT GAA GAC CCA CA & 20 & & \\
\hline MAGE-A3/6 i/s & GGC TCG GTG AGG AGG CAA G & 19 & 148 & 60 \\
\hline MAGE-A3/6 i/as & GAT GAC TCT GGT CAG GGC AA & 20 & & \\
\hline MAGE-A4 in/s & CAC CAA GGA GAA GAT CTG CCT & 21 & 118 & 60 \\
\hline MAGE-A4 in/as & CAG GCT TGC AGT GCT GAC TCT & 21 & & \\
\hline MAGE-A10 in/s & AGG AGG CGG GGG AGG TGA GA & 20 & 80 & 60 \\
\hline MAGE-A10 in/as & GGG TTC CCT GTG TTG ACT TGA G & 22 & & \\
\hline MAGE-A12 in/s & CCG CAG GGA ACT CTG GTA TC & 20 & 63 & 60 \\
\hline MAGE-A12 in/as & ACC TGA GTC ACC CTC TGA AGA AA & 23 & & \\
\hline GAPDH in/s & GAC CCC TTC ATT GAC CTC AAC TA & 23 & 67 & 60 \\
\hline GAPDH in/as & GAA TTT GCC ATG GGT GGA AT & 20 & & \\
\hline
\end{tabular}

cancer development (group 1) was compared to that without cancer development (group 2) by the $\chi^{2}$ test. The association between grade of dysplasia and malignant transformation and MAGE-A expression were analysed by the $\chi^{2}$ or MannWhitney U-test, respectively. P-values $<0.05$ were considered to indicate a statistically significant difference.

\section{Results}

H\&E stained sections of OPL tissues were classified according to the grade of dysplasia by 2 independent pathologists according to the criteria provided by WHO and Warnakulasuriya et al (7). Additionally, the differentiation of tumor specimens and the clinical staging of OSCC arising from OLP were defined.

Clinical features, histopathological data and follow-up of the patients. In this study, 74 OLP samples were examined; 55\% (41/74) were male and $45 \%$ (33/74) female. The average age of the examined patients with OLP was 53.7 years. In total, 38 OLPs had no dysplasia (51.4\%), 26 (35.1\%) showed mild, 7 (9.5\%) moderate and 3 (4\%) severe dysplasia (Fig. 1A). With the increasing level of dysplasia the risk of malignant transformation increases. Out of 38 and 26 OLP exhibiting no or mild dysplasia, 31.6\% (12 samples) and 19.2\% (5 samples) progressed into carcinoma, respectively. By contrast, 57.1\% (4/7) of lesions showing moderate dysplasia and all $3(100 \%)$ OLPs graded as D3 became malignant (Fig. 2). Statistical investigation revealed that the correlation between the risk of tumor development and grade of dysplasia was significant $(\mathrm{p}<0.001)$.

The total contingent was divided into 2 groups. Group 1 included 24 lesions progressing into malignancy within 5 years and group 2 consisted of 50 samples which did not show any malignant transformation (Table II).

Of the 24 progressing OLPs, 15 patients $(63 \%)$ were male and $9(37 \%)$ female. The average age of the examined patients was 60.4 years.

All grades of dysplasia were represented. Among the 24 patients, $12(50 \%)$ had no dysplasia (D0), 5 (21\%) mild, $4(17 \%)$ moderate and $3(12 \%)$ severe dysplasia (Fig. 1B).

Group 2 included 50 OLP tissue samples. Of these, 52\% (26/50) were male and 48\% (24/50) female. The average age of examined patients was 47.2 years. Contrary to group 1 , only samples with D0 to D2 grade were included and no OLP with grade D3 was included. In total, 52\% (26/50) showed no dysplasia (D0), $42 \%$ (21/50) mild and 6\% (3/50) moderate dysplasia. There was no prevalence of D0 or D1 grade of dysplasia within this group. However, samples with a high grade of dysplasia were clearly under-represented (Fig. 1C). 
A

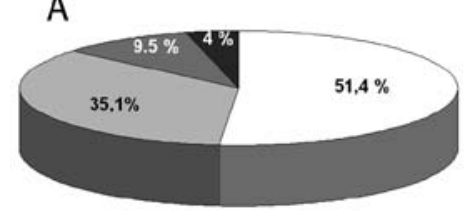

B

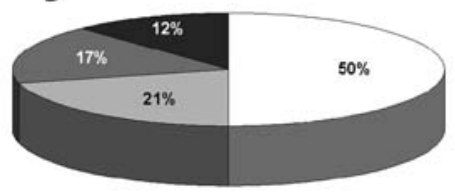

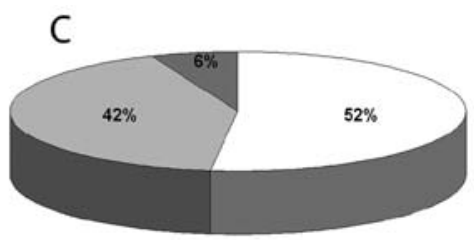

$\mathrm{D} 0 \square \mathrm{D} 1 \square \mathrm{D} 2 \mathrm{\square D} 3$

Figure 1. Distribution of cases of OLP into different grades of dysplasia. (A) Distribution of dysplasia within the total patient cohort. (B) Number of the different grades of dysplasia in progressing OLP: All grades of dysplasia are represented and there is no prevalence of any dysplasia level within this group. (C) Distribution of dysplasia in non-progressing leukoplakia (group 2). Lesions with a high grade of dysplasia are under-represented. There is no prevalence of D0 or D1 lesions.

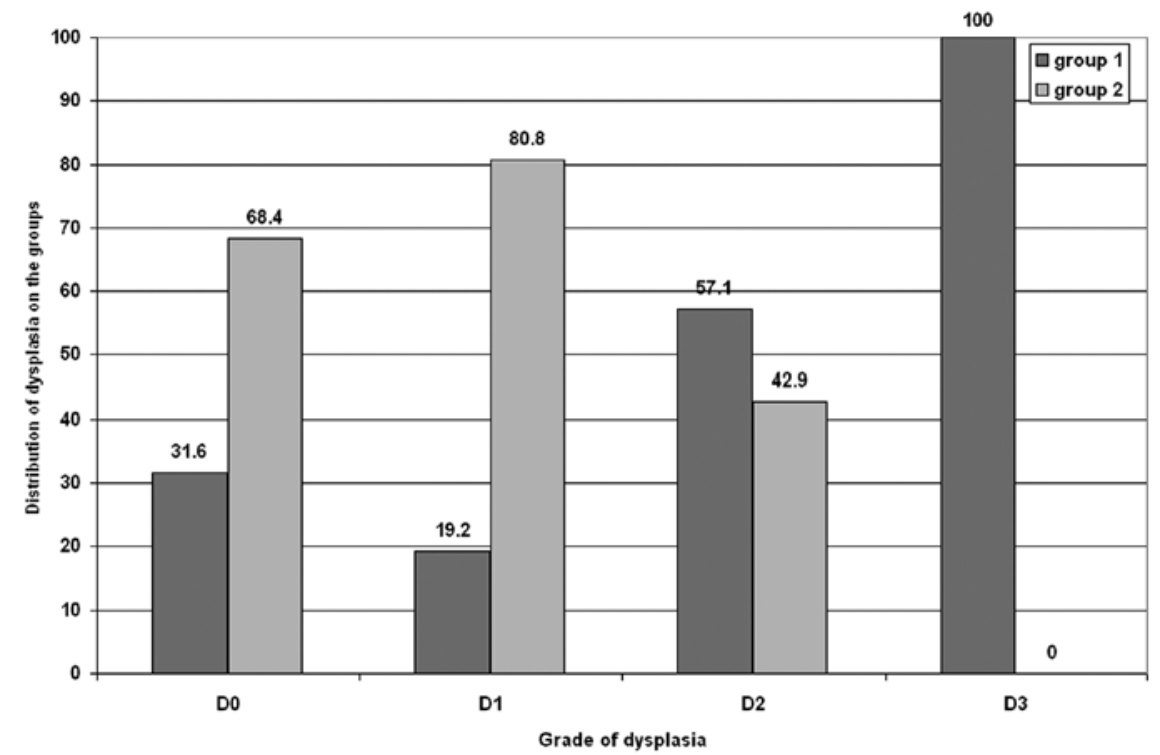

Figure 2. Numbers of malignant transformation relative to the grade of dysplasia. The severity of dysplasia was significantly associated with malignant transformation $(\mathrm{p}<0.05)$. Group 1, progressing OLP; group 2, non-progressing OLP.

$M A G E-A$ expression in progressing and non-progressing $O L P$ and correlation with cancer risk. Representative results from the real-time PCR experiments are shown in Figs. 3 and 4. The results of the expression analyses are summarized in Tables II and III.

In 11 out of the 24 investigated progressive OLPs (46\%) the expression of at least one MAGE-A antigen was detected (Fig. 3 and Table II). Independent of the grade of dysplasia, the samples of the examined non-progressing OLPs expressed no antigen (Fig. 4A). Only 1 lesion showed a very low expression of MAGE-A10 $(\mathrm{Ct}=38)$. Due to the high cycle number, the sample was estimated nevertheless as negative. Statistical analysis revealed that the correlation was significant between MAGE-A expression and malignant transformation $(\mathrm{p}=0.0001)$.

The distribution of MAGE-A expression was defined with regard to the grade of dysplasia within progressive OLPs. Out of 12 lesions graded as D0, 5 (42\%) were positive. The expression frequency in lesions exhibiting moderate and severe dysplasia was approximately $100 \%(4 / 4)$ and $67 \%(2 / 3)$, respectively (Table III). No statistically relevant correlation between the dysplasia of the progressing lesion, staining and the potential of malignant transformation was found $(\mathrm{p}=0.54)$.
The time interval between the diagnosis of OLP and the detection of malignancy (DFS) was between 1 to 60 months. The mean DFS was 19.5 months and varied between 17.4 and 21 months within the different stages of dysplasia (D0, 17.4; D1, 19.3; D2, 20.5; D3, 25 months). The DFS in the stained specimens was lower (14.8 months) than in the unstained group (23.5 months), but this difference was not statistically significant $(\mathrm{p}=0.207)$.

Clinical and histological characterization of corresponding OSCC and expression of MAGE-A antigens in malignant and normal mucosa. Most of the 24 corresponding OSCC tumors were small at the time of diagnosis. Of these, 17 were classified as T1 (70.8), 5 as T2 (20.8\%), none as T3 $(0 \%)$ and 1 as T4 (4.2\%) tumors. In 1 case, a carcinoma in situ (CIS) was diagnosed (4.2\%). Most patients did not suffer from lymph node metastasis. Out of the 17 classified cases, the lymph node was not affected in 14 patients $(\mathrm{N}=0 ; 82.3 \%)$. In 3 patients lymph node metastases was diagnosed $(\mathrm{N}>0 ; 17.6 \%)$. In total, 87.5 $\%$ of the patients (21/24) developing a malignancy were diagnosed during the early stages of the disease. Only $12.5 \%$ of the patients had the late stages of the disease. All OSCCs except 

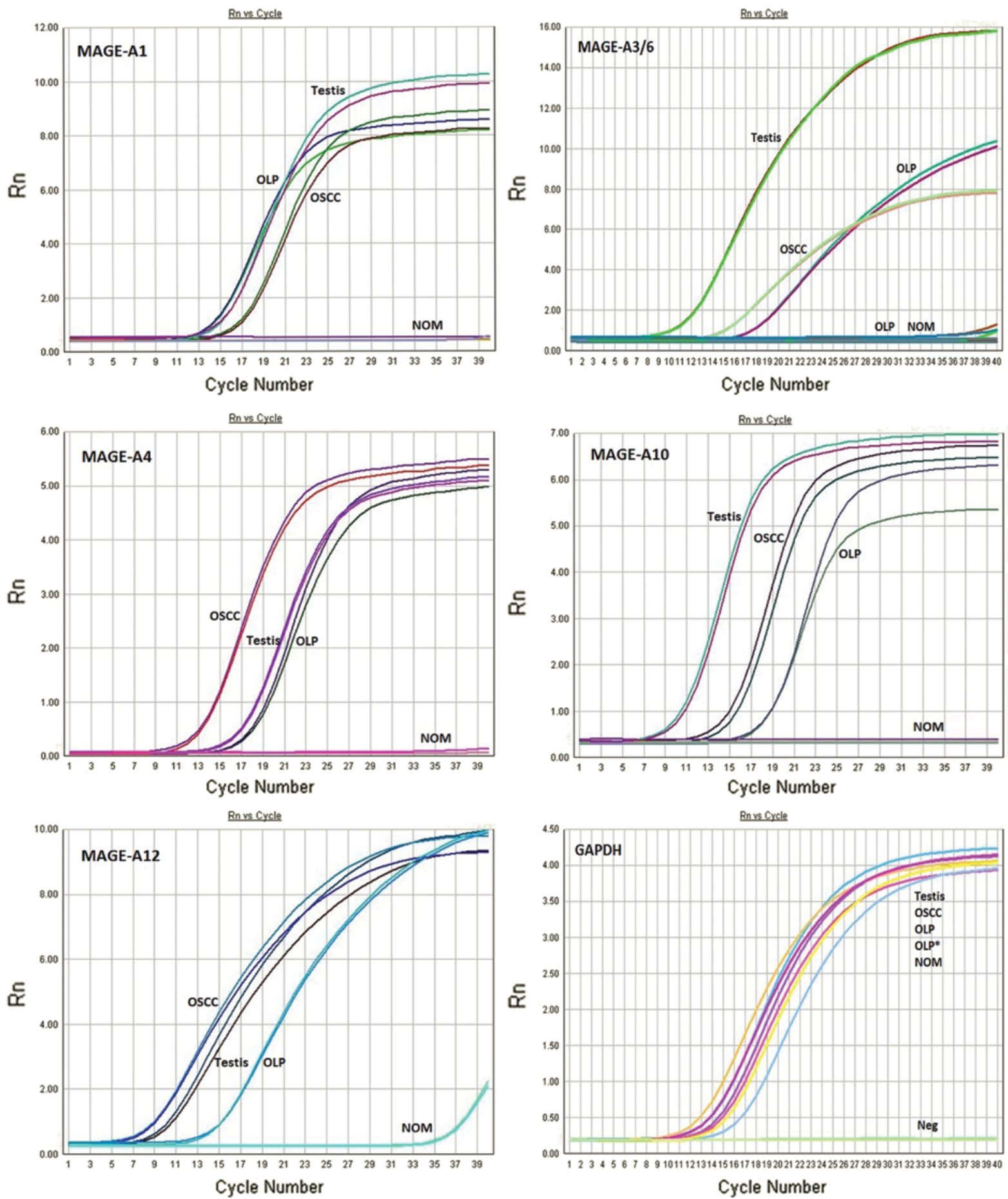

Figure 3. Expression analysis of progressing OLP. Expression of the different examined members of the MAGE-A family in testis, OLP and in the corresponding malignant lesion. Normal oral mucosa served as the negative control. The quality of RNA was assessed by amplifying cDNA using GAPDH as the internal control. OLP, oral leukoplakia; OLP*, negative leukoplakia; OSCC, corresponding oral squamous cell carcinoma; NOM, normal oral mucosa (false products identified by dissociation curves). Neg, negative control reaction without cDNA template.

CIS were graded for differentiation. In total, 9 (39.1\%) OSCCs were well-differentiated (G1), 8 (34.8\%) were moderately (G2) and $6(26.1 \%)$ were poorly differentiated.

For the 18 OSCC samples, MAGE- A expression analyses was performed. In total, 13 specimens expressed at least 1 antigen (72.2\%; Table II). All OSCCs based on positive OLPs expressed the same antigens as the pre-cancerous lesion. A total of 5 negative OLPa developed into an antigen-expressing malignancy.
All 30 specimens of normal oral mucosa from healthy volunteers did not show remarkable changes, such as inflammation and hyper/dysplasia, or any other clinical abnormalities. MAGE-A expression was not observed in any of these tissues (Fig. 4B and Table II). The expression was restricted to malignant lesions and allowed the distinction between healthy and malignant tissues. Additionally, the correlation between malignancy and MAGE-A expression was statistically significant $(\mathrm{p}=0.0001)$. 
A

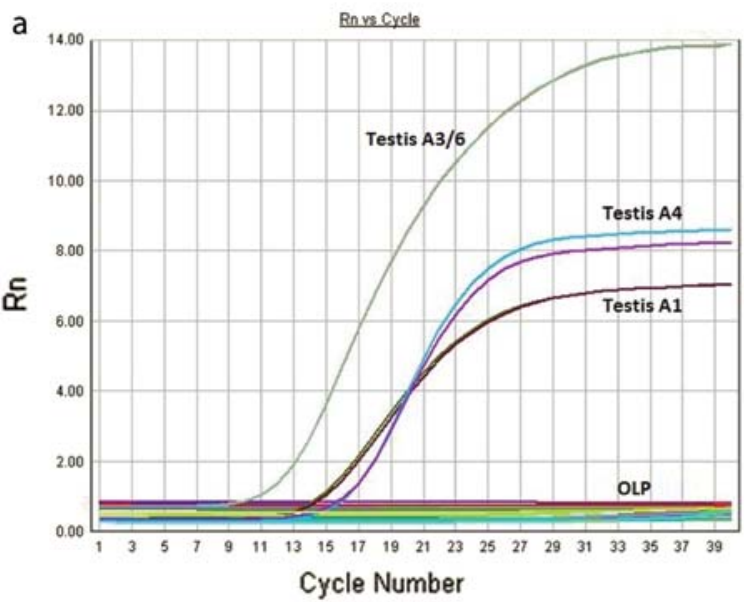

B

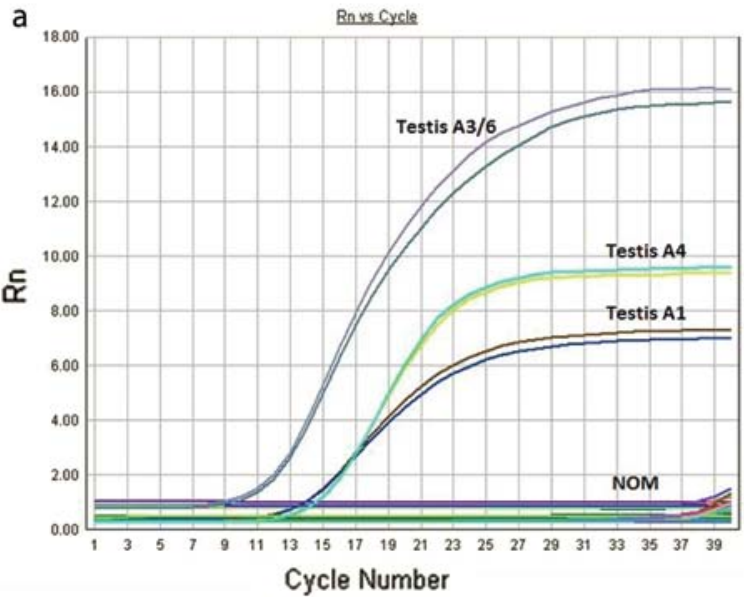

b

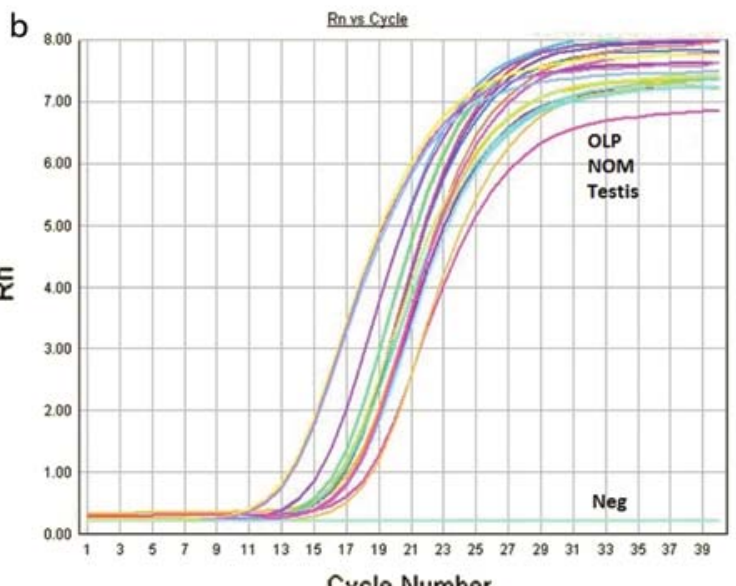

Cycle Number

b

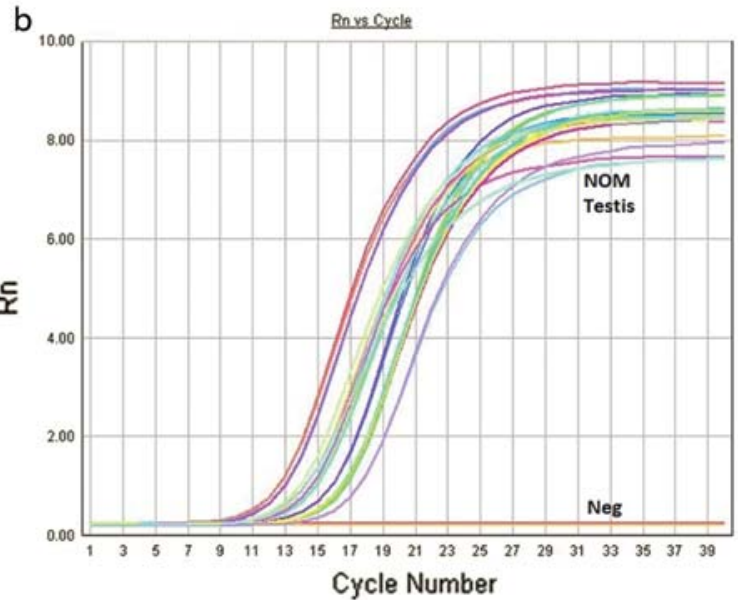

Figure 4. Expression analysis in non-progressing OLP and in tissues of normal oral mucosa. (A) Expression analysis in non-progressing OLP. (a) Expression analyses of MAGE-A 1, 3/6 and 4 in testis (positive control) and 10 non-progressing OLP. (b) The quality of RNA was assessed by amplifying cDNA using GAPDH as the internal control. (B) Expression analysis in normal oral mucosa. (a) Expression analyses of MAGE-A 1, 3/6 and 4 in testis (positive control) and 10 normal oral mucosa specimens. (b) The quality of RNA was assessed by amplifying cDNA using GAPDH-as the internal control. Neg, negative control reaction without cDNA template.

Table II. MAGE-A expression in different types of tissues.

\begin{tabular}{lrrcc}
\hline Tissue & $\begin{array}{c}\text { No. } \\
\text { of cases }\end{array}$ & + & $\begin{array}{c}\text { Positive } \\
(\%)\end{array}$ & $\begin{array}{l}\text { p-value } \\
\left(\chi^{2} \text { test }\right)\end{array}$ \\
\hline Progressing OLP & 24 & 11 & 46 & $0.00001^{\mathrm{b}}$ \\
Non-progressing OLP $^{\mathrm{C}}$ & 50 & 0 & 0 & \\
Corresponding tumor $^{\mathrm{a}}$ & 18 & 13 & 72.2 & $0.00001^{\mathrm{c}}$ \\
Normal oral mucosa $^{\mathrm{c}}$ & 30 & 0 & 0 & \\
\hline
\end{tabular}

Antigen expression was only detected in progressive and malignant tissues. ${ }^{a}$ All OSCC based on positive OLPs retained antigen expression. ${ }^{\mathrm{b}} \mathrm{High}$ cancer risk and ${ }^{\mathrm{c}}$ diagnosis significantly correlated with antigen detection.

\section{Discussion}

Not all OSCCs develop de novo on healthy mucosal tissue, but rather result from the transformation of several oral potentially pre-cancerous lesions (28). The early diagnosis of OSCC and the identification of high-risk precursors play a key role in patient survival and the incidence of OSCC. OLP is the most
Table III. MAGE-A staining within progressive leukoplakia.

\begin{tabular}{lcrr}
\hline $\begin{array}{l}\text { Grade of } \\
\text { dysplasia }\end{array}$ & No. of samples & + & $\begin{array}{c}\text { Positive } \\
(\%)\end{array}$ \\
\hline Total & 24 & 11 & 46 \\
D0 & 12 & 5 & 42 \\
D1 & 5 & 0 & 0 \\
D2 & 4 & 4 & 100 \\
D3 & 3 & 2 & 67
\end{tabular}

The grade of dysplasia of OLP did not significantly correlate with MAGE-A expression and malignant transformation $(\mathrm{p}=0.54)$.

common oral potentially pre-cancerous lesion, accounting for over $80 \%$ of the potentially malignant oral disorders. Additionally, it has been reported that between 11 and $67 \%$ of all OSCCs are based on OLPs, demonstrating a significant increase in the incidence of OSSCs arising from OLPs $(10,29)$. The evaluation of an asymptomatic patient for earlystage cancer, based on physical features alone, is frequently 
compromised, as malignant and benign lesions may not be clinically distinguishable (30). Currently, the risk assessment of OLP is performed based on histological staining and the determination of dysplasia defined as mild, moderate or severe dysplasia, whereby the risk of the malignant transformation rises with the severity $(7,8)$. However, this method lacks objectivity and sensitivity within the whole lesion. Furthermore, the histological features cannot accurately predict the outcome of individual cases. Thus, it is possible that individual moderate and severe dysplasia remain stable or regress, whereas lesions without dysplasia or mild dysplasia will progress to malignancy $(6-8,10,16)$. These problems call for the identification of reliable molecular biomarkers to identify individuals with potentially malignant disorders who are at a high risk of developing OSCC $(10,14,16,31)$.

Alternations in genes and pathways that regulate cellular signalling, cell cycle, differentiation, apoptosis, genomic stability and angiogenesis are significantly associated with the progression of a potentially malignant disorder to OSCC and have been considered as biomarkers for cancer risk assessment. However, these biomarkers have not gained any use in routine diagnosis and risk assessment either alone or in combination. Additionally, in almost all studies, the expression was only associated with the grade of dysplasia and follow-up was rarely carried out $(16,31)$. Therefore, only an additional aid in the evaluation of the severity of OLP was shown, and the importance of these markers in forecasting the incidence of the disease remains unknown. Hence, identifying further diagnostic tools remains crucial for receiving the most representative analysis of any suspicious lesions.

The expression of MAGE-A is specific for malignancies, including OSCC and discrimination between healthy mucosa and tumor tissues is possible with high accuracy and probability (21-23). Recently, MAGE-A expression was shown in OLP. However, whether the detection of MAGE-A expression accurately predicts malignant transformation with time, has not been elucidated, as previous studies have included only a small number of cases of individual oral tissue samples and/ or the clinical course of the disease has not been assessed (24-26). In our retrospective study, we compared the expression of MAGE-A between patients with OLP, who did or did not develop OSCC within 5 years. Despite the pre-selection of the samples met on their clinical and biological behaviour, a statistically significant association between the severity of dysplasia and malignant transformation was demonstrated. However, within progressing leukoplakia all grades of dysplasia were represented. This indicates that cancer risk also emanates from non- and mild dysplastic lesions, although with a small probability.

MAGE-A expression was only detected in progressive OLP. All stages of dysplasia and even lesions without histopathological changes were represented. If the expression of the marker was detected in the lesion, an OSCC arose at the appropriate anatomical region within a period of 5 years in $46 \%$ of all cases. The correlation between the detection of MAGE-A in OLP and malignant transformation was highly significant. Thus, high-risk patients can be identified with high accuracy and probability. Furthermore, no significant association between MAGE-A expression and grade of dysplasia was found within the progressive group. Hence, the malignant potential can be estimated independently of the grade of dysplasia. Additionally, the expression of MAGE-A by non dysplastic lesions which progressed into carcinoma is reported for the first time indicating that their malignant potential can be assessed by the expression analyses of MAGE-A genes. Thus, high-risk or already transformed lesions which are not dangerously classified by histopathological investigation can also be identified. This shows the efficacy of this method for the risk assessment of D0 lesions. This method may be a reliable additional molecular marker for the prediction of malignant transformation and may help to differentiate harmless and high-risk lesions. However, although multiple gene expression analysis was applied, the expression frequency was too low for sensitive diagnostic screening. Therefore, the method is strongly recommended in conjunction with additional markers for malignant progression in order to increase sensitivity. Therefore the identification of additional reproducible molecular markers by multi-centre studies with larger cohorts for multivariate analyses is urgently required.

Epigenetic modifications, most notably changes in the global DNA methylation level, are a hallmark in cancer genesis and tumor progression and occur also during tumorigenesis of OSCC $(32,33)$. The precise regulatory mechanism of MAGE-A expression is still not fully understood, but it has previously been demonstrated to be linked to overall DNA demethylation $(34,35)$. Therefore, it is accepted that the repression of these genes is an early step within oral carcinogenesis which may pinpoint the malignant transformation. These facts confirm the results that MAGE-A expression in oral lesions may be a considerably early diagnostic tool. Furthermore, OSCCs based on positive OLPs show MAGE-A expression. Thus residual cells descending from a preceding positive lesion can be proven during tumor development and progression. This could allow for the early identification of recurrences based on remaining residual cells of the original OLP. Moreover, the expression frequency in OSCC samples is higher than in precursor lesions. Previous studies have demonstrated the high expression frequencies of MAGE-A expression in OSCC $(23,36,37)$. Thus, MAGE-A expression increases with the progression of malignancy by demethylation mechanism.

Our results may also have an impact on the clinical management of OLP. Treatment currently consists of excision, laser treatment or watchful waiting. Depending on the presence of epithelial dysplasia, the frequency of the patient follow-up varies (8). In our study, the mean DFS was almost equal in all the groups of dysplasia, independent of the staining result. Thus, it may prove beneficial to apply small observation periods to all patients. Nevertheless, for patients with MAGE-A-expressing OLPs more radical treatment has to be chosen, as all positive OLPs manifested themselves promptly. Thus, positive lesions with limited dimensions can always be excised with larger surgical margins and for patients with low-risk lesions, a good follow-up would suffice. It is expected that cancer incidence, patient survival rate and quality of life would considerably increase, as planning the best treatment modality will be assisted by an objective additional tool leading to earlier, more adequate and even more radical treatment of the dangerous lesion.

However, due to the widespread damage of certain tissues and the multifocal development of lesions, traditional therapy 
is not always possible. Hence, the development of better therapeutic strategies to delay or reverse incidences of malignant progression is urgently required. Most of the new therapeutic strategies demonstrate the immunotherapeutic approach. Due to their restricted expression to malignant cells and testis and their high immunogenetic effect, MAGE-A antigens are possibly ideal targets for immunotherapy $(36,38-40)$. The high expression frequency of MAGE-A in progressive OLP may also recommend these proteins as a potential targets for the antigen-specific immunotherapy and polyvaccination in precancerous lesions.

Additionally, less invasive methods may be established. One example is the expression analyses in samples obtained by the cytobrush method (41). The exfoliate cytology using a cytobrush is minimally invasive, painless and allows multiple sampling from different parts of the lesion of larger areas and of multifocal lesions. Therefore, it can be used effectively and routinely to follow-up suspected oral lesions as well as resected tumour cases with a high recurrence rate. Additionally, the problem of the limited reproducibility of incision biopsies within the whole lesion may be solved. Recent studies already point to the possible application of the combination of the cytobrush method and MAGE-A expression analyses in the early diagnoses of OSCC, risk assessment of OLP and the detection of possible early recurrences by close follow-up after surgery $(25,42)$. This tool may help to increase the patient survival rate and quality of life. However, these studies include only a few samples and further investigations on a larger patient contingent are required to confirm the impact of this method.

In conclusion, the expression analysis of multiple MAGE-A genes might be a reliable additional molecular diagnostic marker to predict the malignant transformation of OLP independently of the ascertained grade of dysplasia. Moreover, non-dysplastic OLPs presenting a high risk of cancer could be identified and individually treated. Furthermore, alternative diagnostic procedures, such as brush biopsy and new therapeutic procedures, notably immunotherapeutic approaches, may be established. These improvements may decrease the incidence of the disease and increase the 5-year disease survival rate by adequate and earlier treatment of both highrisk lesions and OSCC.

\section{Acknowledgements}

This study was supported by the ELAN-Fonds of the University of Erlangen and Deutsche Forschungsgemeinschaft (DFG). The authors would also like to thank Ms. A. Krautheim-Zenk, Ms. S. Schönherr and Ms. E. Diebel for their valuable technical support.

\section{References}

1. Jemal A, Siegel R, Xu J and Ward E: Cancer statistics, 2010. CA Cancer J Clin 60: 277-300, 2010.

2. Lung T, Tascau OC, Almasan HA and Mureşan O: Head and neck cancer, treatment, evolution and post therapeutic survival Part 2: a decade's results 1993-2002. J Craniomaxillofac Surg 35 126-131, 2007.

3. Gondos A, Arndt V, Holleczek B, et al: Cancer survival in Germany and the United States at the beginning of the 21st century: an up-to-date comparison by period analysis. Int $\mathrm{J}$ Cancer 121: 395-400, 2007.
4. Hunter KD, Parkinson EK and Harrison PR: Profiling early head and neck cancer. Nat Rev Cancer 5: 127-135, 2005.

5. Warnakulasuriya S, Johnson NW and van der Waal I: Nomenclature and classification of potentially malignant disorders of the mucosa. J Oral Pathol Med 36: 575-580, 2007.

6. Fleskens S and Slootweg P: Grading systems in head and neck dysplasia: their prognostic value, weaknesses and utility. Head Neck Oncol 1: 11-18, 2009.

7. Warnakulasuriya S, Reibel J, Bouquot J and Dabelsteen E: Oral epithelial dysplasia classification systems: predictive value, utility, weaknesses and scope for improvement. J Oral Pathol Med 37: 127-133, 2008.

8. Van der Waal I: Potentially malignant disorders of the oral and oropharyngeal mucosa; terminology, classification and present concepts of management. Oral Oncol 45: 317-323, 2009.

9. Pindborg JJ, Reichart PA, Smith CJ and van der Waal I: World Health Organization: Histological Typing of Cancer and Precancer of the Oral Mucosa. Vol. 14. Springer-Verlag, Berlin, pp47-62, 1997.

10. Reibel J: Prognosis of oral pre-malignant lesions: significance of clinical, histopathological, and molecular biological characteristics. Crit Rev Oral Biol Med 14: 47-62, 2003.

11. Holmstrup P, Vedtofte P, Reibel J and Stoltze K: Oral premalignant lesions: is a biopsy reliable? J Oral Pathol Med 36: 262-266, 2007.

12. Fleskens SA, Bergshoeff VE, Voogd AC, et al: Interobserver variability of laryngeal mucosal premalignant lesions: a histopathological evaluation. Mod Pathol 24: 892-898, 2011.

13. Brennan M, Migliorati CA, Lockhart PB, et al: Management of oral epithelial dysplasia: a review. Oral Surg Oral Med Oral Pathol Oral Radiol Endod 103 (Suppl 19): e1-e12, 2007.

14. Warnakulasuriya S: Lack of molecular markers to predict malignant potential of oral precancer. J Pathol 190: 407-409, 2000.

15. Ha PK, Chang SS, Glazer CA, et al: Molecular techniques and genetic alterations in head and neck cancer. Oral Oncol 45: 335-339, 2009.

16. Pitiyage G, Tilakaratne WM, Tavassoli M and Warnakulasuriya S: Molecular markers in oral epithelial dysplasia: review. J Oral Pathol Med 38: 737-752, 2009.

17. Nasser W, Flechtenmacher C, Holzinger D, et al: Aberrant expression of p53, p16(INK4a) and Ki-67 as basic biomarker for malignant progression of oral leukoplakias. J Oral Pathol Med 40: 629-635, 2011

18. Vairaktaris E, Yapijakis C, Derka S, et al: Association of platelet glycoprotein Ia polymorphism with minor increase of risk for oral cancer. Eur J Surg Oncol 32: 455-457, 2006.

19. Vairaktaris E, Yapijakis C, Yiannopoulos A, et al: Strong association of the tissue inhibitor of metalloproteinase-2 polymorphism with an increased risk of oral squamous cell carcinoma in Europeans. Oncol Rep 17: 963-968, 2007.

20. Yapijakis C, Vairaktaris E, Vassiliou S, et al: The low VEGF production allele of the $+936 \mathrm{C} / \mathrm{T}$ polymorphism is strongly associated with increased risk for oral cancer. J Cancer Res Clin Oncol 133: 787-791, 2007

21. Jungbluth AA, Busam KJ, Kolb D, et al: Expression of MAGEantigens in normal tissues and cancer. Int J Cancer 85: 460-465, 2000.

22. Muller-Richter UD, Dowejko A, Peters S, et al: MAGE-A antigens in patients with primary oral squamous cell carcinoma. Clin Oral Investig 14: 291-296, 2010.

23. Ries J, Mollaoglu N, Toyoshima T, et al: A novel multiple-marker method for the early diagnosis of oral squamous cell carcinoma. Dis Markers 27: 75-84, 2009.

24. Krauss E, Rauthe S, Gattenlohner S, et al: MAGE-A antigens in lesions of the oral mucosa. Clin Oral Investig 15: 315-320, 2011.

25. Metzler P, Mollaoglu N, Schwarz S, et al: MAGE-A as a novel approach in the diagnostic accuracy of oral squamous cell cancer: a case report. Head Neck Oncol 39: 1-6, 2009.

26. Ries J, Ponader S, Mollaoglu N, et al: Early findings of a novel established molecular diagnostic technique for the prediction of malignant transformation in leukoplakia. Mol Med Rep 2: 947-952, 2009.

27. Mecklenburg I, Weckermann D, Zippelius A, et al: A multimarker real-time RT-PCR for MAGE-A gene expression allows sensitive detection and quantification of the minimal systemic tumor load in patients with localized cancer. J Immunol Methods 323: 180-193, 2007.

28. Scheifele $\mathrm{C}$ and Reichart PA: Is there a natural limit of the transformation rate of oral leukoplakia? Oral Oncol 39: 470-475, 2003. 
29. Scheifele $\mathrm{C}$ and Reichart PA: Oral leukoplakia in manifest squamous epithelial carcinoma. A clinical prospective study of 101 patients. Mund Kiefer Gesichtschir 2: 326-330, 1998 (In German)

30. Rethman MP, Carpenter W, Cohen EE, et al: Evidence-based clinical recommendations regarding screening for oral squamous cell carcinomas. J Am Dent Assoc 141: 509-520, 2010.

31. Oliveira LR and Ribeiro-Silva A: Prognostic significance of immunohistochemical biomarkers in oral squamous cell carcinoma. Int J Oral Maxillofac Surg 40: 298-307, 2011.

32. Ehrlich M: DNA methylation in cancer: too much, but also too little. Oncogene 21: 5400-5413, 2002.

33. Diez-PerezR,Campo-Trapero J, Cano-Sanchez J, et al: Methylation in oral cancer and pre-cancerous lesions (Review). Oncol Rep 25: 1203-1209, 2011.

34. Simpson AJ, Caballero OL, Jungbluth A, et al: Cancer/testis antigens, gametogenesis and cancer. Nat Rev Cancer 5: 615-625, 2005.

35. De Smet C,Loriot A and Boon T: Promoter-dependent mechanism leading to selective hypomethylation within the 5 ' region of gene MAGE-A1 in tumor cells. Mol Cell Biol 24: 4781-4790, 2004.

36. Ries J, Schultze-Mosgau S, Neukam F, et al: Investigation of the expression of melanoma antigen-encoding genes (MAGE-A1 to -A6) in oral squamous cell carcinomas to determine potential targets for gene-based cancer immunotherapy. Int J Oncol 26 $817-824,2005$
37. Ries J, Vairaktaris E, Mollaoglu N, et al: Expression of melanoma-associated antigens in oral squamous cell carcinoma. J Oral Pathol Med 37: 88-93, 2008.

38. Sang M, Lian Y, Zhou X and Shan B: MAGE-A family: Attractive targets for cancer immunotherapy. Vaccine 29: 8496-8500, 2011.

39. Atanackovic D, Blum I, Cao Y, et al: Expression of cancer-testis antigens as possible targets for antigen-specific immunotherapy in head and neck squamous cell carcinoma. Cancer Biol Ther 5: 1218-1225, 2006.

40. Rapidis AD and Wolf GT: Immunotherapy of head and neck cancer: current and future considerations. J Oncol 2009: 346345, 2009.

41. Lingen MW, Kalmar JR, Karrison T and Speight PM: Critical evaluation of diagnostic aids for the detection of oral cancer. Oral Oncol 44: 10-22, 2008.

42. Mollaoglu N, Metzler P, Zenk J, et al: Prediction of recurrence using exfoliative cytology and melanoma-associated antigen-A mRNA analysis following wide excision of oral squamous cell carcinoma: short report. Cytopathology 22: 387-391, 2011. 